\title{
29. New Rat Astrocytoma Cell Lines induced in vitro by Chemical Adhesive of Epoxy Resin-Polyamine Composition
}

\author{
By Kyoko Kano-TanaKa,*),**) Taiji Kato,***) Jin-ichi Ito,***) \\ Ryo TANAKA,***) Hiroko FuKami,*) Shigeo OKUMURA,****) \\ and Tatsuya TANAKA*),**)
}

(Communicated by Setsuro Ebashi, M. J. A., March 12, 1986)

Introduction. An adhesive concerned in the present study is a synthetic resin-type one which is commercially available in 2 solutions, the main materials made of uncured epoxy resin and the reactive hardner made of polyamine. There is no evidences so far indicated that this adhesive mixture is tumorigenic. We actually used it to adhere a silicon bar on the bottom of plastic culture dishes for the purpose of making wells on the dish. The dishes thus prepared, however, were found to exert severe cytotoxic effects on cultured rat glioblasts. It was an unexpected event, therefore, that cells surviving in culture underwent rapid malignant transformation. The present information deals with the process of malignant transformation and characteristics of the isolated cell lines as astroglial cells.

Materials and methods. The procedure for preparation of the primary culture of glioblasts has been described. ${ }^{7}$ ) The brain cells in the primary culture were then subcultured into the $60 \mathrm{~mm}$ plastic dish (Falcon Co.) with 2 wells divided by Silicon bar which was adhered at the bottom by an epoxy resin-type of adhesive, for the purpose of some neurological experiments. The dishes thus prepared have been sterilized by either ethylene oxide gas (Dish A) or ethanol (Dish B). Culture media used after the secondary culture passages were either Ham's F-10 or Dulbecco's modification of Eagle's minimal essential medium (DMEM, GIBCO) with 10\% FCS. To determine whether any of the 2 solutions of the adhesive, uncured epoxy resin (ER) and polyamine (P) possesses transformability, stock solutions were prepared by adding the substances $(1 \mathrm{gm} / \mathrm{ml})$ in culture medium, kept overnight, and sterilized by filtration. Spermine (synthesized by S. Okumura) was dissolved $1 \mathrm{M}$ in ethanol as the stock solution. These were diluted to final concentration in medium immediately before use. The exposure to each agent was initiated with $10^{6}$ cells per dish at the secondary culture and continued for about one month by changing the medium every 3 to 5 days. Transformed cells were tested for the ability to form colonies in soft agar $(0.5 \mathrm{ml}$ of $0.3 \%$ Sea Plaque Agarose in DMEM with $10 \%$ FCS, on a base layer of $3 \mathrm{ml}$ of $0.6 \%$ agar in the same medium) or transplanted with $10^{7}$ cells into syngeneic sucklings or weanlings.

464.

*) Laboratory of Cell Biology, Aichi Cancer Center Research Institute, Nagoya

**) Division of Neurobiology, Awazu Neuro-Sanatorium, Gamagori 443-01.

***) Department of Biochemistry, Nagoya City University Medical School, Nagoya 467.

****) Laboratory of Biochemistry, Aichi Medical University, Nagakute 480-11, Japan. 
Results. Normal glioblasts initially grow as a monolayer sheet of epithelioid cells. At the subsequent passages, various types of differentiated glial cells such as astrocyte and oligodendrocyte appeared on the top of the monolayer, and formed in some cases small colonies. After 1 to 2 months, however, the cells tended to be dominated by again monolayer cells which are morphologically different from glioblasts (more astrocytic) and gradually lost growth ability. Some of these cultures could survive for nearly 6 months, although all untreated cells died eventually. None of the control cultures underwent spontaneous malignant transformation.

When the glioblasts of Wistar rats were plated on the Dish A exhibited severe cytotoxic damages. These dishes were repeatedly rinsed with Tyrode solution in order to remove all the cell debris, added Tyrode solution and kept for 7 days. Freshly prepared primary glioblasts were then seeded into four recleaned dishes. The only areas along with adhered Silicon bar still retained to exert cytotoxic effects on cells. About 2 weeks later, however, all dishes began to show the active proliferation of survived cells in forming many colonies. About a month later, more rapid proliferation of morphologically altered cells spreading on the flat epithelioid cell layer was observed. These cells readily became continuous cell lines, and one clonal line termed GA1, was maintained.

Although this case was rather an unexpected event, it was remarkable that glioblasts exposed to the adhesive underwent marked and extremely rapid changes resulting in the expression of malignancy in vitro. Experiments were repeated with BDIX rat glioblasts. A similar pattern of malignant transformation was observed in one of the experimental dishes after the cultivation for 4 weeks. The transformants grown in soft agar were then cloned to establish a cell line GA2. Both GA1 and GA2 formed malignant tumors which histologically resemble astroglioma in their respective hosts with a similar latent period (about 2 weeks). The outline of experimental sequence is illustrated in Fig. 1. High activities of marker enzymes such as CNPase and GPDH in these cell lines are charac-

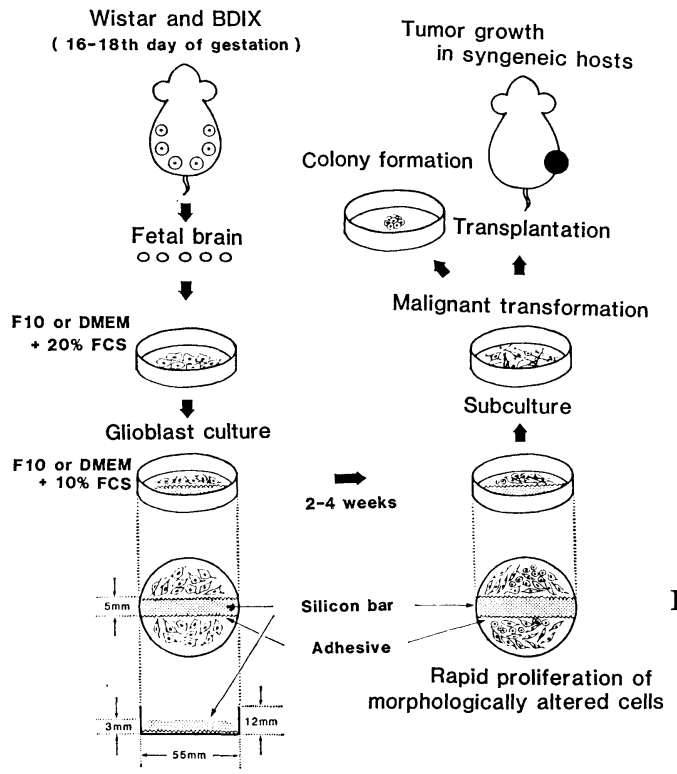

Fig. 1. Diagramatic representation of experimental sequence of malignant transformation of rat glial cells exposed to an adhesive. 
Table I. Glial marker proteins and enzymes in the extract of glial cells

\begin{tabular}{lrrr}
\hline & GA-1 & GA-2 & \multicolumn{1}{c}{ C6 } \\
\hline S-100 protein (ng/mg prot.)*2 & 260.4 & 235.8 & 379.6 \\
Glia fibrillary acidic protein ( $\mu$ unit/mg prot.)*2 & 4.6 & 9.4 & 10.5 \\
$\alpha$-enolase $\left(\mu \mathrm{g} / \mathrm{mg}\right.$ prot.) ${ }^{* 2}$ & 1.88 & 3.64 & 1.58 \\
\hline $2^{\prime}: 3^{\prime}$ cyclic nucleotide phosphohydrolase ${ }^{* 3}$ & 182 & 250 & $172^{* 1}$ \\
$\quad($ nmol NADPred/min/mg prot.) & & & \\
$\quad \begin{array}{l}\text { Glycerolphosphate dehydrogenase } \\
\quad \text { (m unit/min/mg prot.) }\end{array}$ & 834 & 318 & $180 * 1$ \\
\hline
\end{tabular}

*1 Data from Kato, T. et al. ${ }^{6}$ ) Other data indicate the mean values from duplicate or quadruplicate experiments. $\quad * 2$ Kato, K. et al. ${ }^{6)} \quad * 3$ Sojin, D. C. ${ }^{12)}$ *4 White, H. B., III and Kaplan, N. ${ }^{13)}$

teristic of malignant glial cells. The intracellular contents of glial marker proteins (S-100 protein, GFA and $\alpha$-enolase) which are also characterized as astroglioma cells, were comparably high to those of $\mathrm{C} 6$ astrocytoma cells (Table I). Karyotypic analyses of both lines of cell showed some differences in their stem lines (43, XY;2q-, 4q+, +12 for GA1 and normal diploid 42, XY for GA2). Several types of chromosome abnormalities observed in GA cells were, however, identical with those of virally or chemically transformed glial cells and $\mathrm{C} 6$ cells. ${ }^{4), \pi)}$ Details will be described separately.

In order to determine whether the possible effective agent is produced by the adhesive after reacting with ethylene oxide gas, Dish B was tested for toxicity. No cytotoxic effects on glioblasts could be demonstrated. Since Dish B was treated with ethanol, instead of ethylene oxide gas, it was assumed that the causative agent must be alcohol-soluble. Subsequently, the solution $(\mathrm{P})$ but not solution (ER) was found to be alcohol-soluble. When glioblasts were exposed to the solution (P) for several days exhibited dose-dependent cytotoxic changes. A hundred-fold dilution of this stock solution caused as much as $70 \%$ toxicity.

Findings from the above experiments suggested that polyamine may be responsible for the induction of malignant transformation. We ascertained that the solution $(\mathrm{P})$ was trimethylene-tetramine which is related to spermine in structural formula. The similar cytotoxic effects to those by solution (P) were obtained from the BDIX glioblast cultures at over $20 \mu \mathrm{M}$ of spermine whereas no effects at under $10 \mu \mathrm{M}$. The morphologically unaffected cells were cultivated for a month in the presence of spermine by replacing with fresh medium, and then subcultured.

Although the maintenance of these culture was rather difficult, one of the treated cell passages which has been forming small colonies of normal-looking cells started to proliferate after about 5 months of cultivation, and subsequently exhibited active growth of phenotypically altered cells with normal karyotype. These cells showed GA cell-like morphology during in the logarithmic phase of their growth but became fibroblastic as reach to confluence. This line was designated GA7. Attempts to produce tumor in vivo are so far unsuccessful.

Discussion. Malignant transformation of rat brain cells in an "in vivo-in vitro system" has been established ${ }^{9)}$ : When ethylnitrosourea pretreated brains from rat fetuses were transferred to cell culture, malignant transformation took place in brain cells after 200 days or so. On the other hand, when rat glioblasts were exposed to retrovirus in vitro, the induction of malignant transformation 
was obtained within 1 to 3 months. ${ }^{4}$ The present results indicated the rapid malignant changes of rat glial cell in vitro. GA cell lines isolated herein appeared to show similar growth pattern and morphological and biochemical characteristics to the above malignant cells as well as $\mathrm{C} 6$, a conventional astrocytoma clone. ${ }^{8}$ These cell lines may therefore also provide useful model for astrocytomas in biochemical research.

At the moment, the active principle of the adhesive mixture used here is unknown. An uncured epoxy resin as the composition of the adhesive is a liquid aromatic compound with low molecular weight which is most probable to be identical to epoxy resin B1 designated by Hine et al..$^{3}$ ) They have evaluated that this compound is no or extremely low potent carcinogen based on the study of tumor production in mice, rats and rabbits. On the other hand, no experimental evidence has been available for tumorigenicity of any polyamines. The present results suggested the possible attribution of the hardner (polyamine), but not of epoxy resin to a transformation mechanism by the adhesive. Generally, polyamines are known to be normal cell constituents that play an important role in cell proliferation and differentiation. They bind strongly to DNA in vitro. ${ }^{1}$ Recent studies on physiological functions by them in animals also indicated their possible interactions with nucleic acids. ${ }^{11)}$ It may be therefore quite reasonable that the exogenous polyamine exerts severe cytotoxic effects at high dosages whereas it induces genic changes at low dosages. Gfiller et al. ${ }^{2)}$ have observed nucleolar changes after in vitro isolation of rat liver nucleus in the presence of polyamines, the most prominent changes by spermine, and assumed that the effects on DNA might be specific for the nucleolar organizer. If the hardner indeed participated in adhesive-induced transformation as a causative agent, such an assumption is interested in that it brings about some ideas of the possible mode of action. The present tests with spermine demonstrated no malignant but morphological transformation and persistent growth of glial cells although whether or not they are preneoplastic could not be identified. In the case of the adhesive, however, we should also consider a possibility of any participation of free radicals produced by breakdown of epoxy polymers as an another tumorigenic agent.10) Further, it is likely that such a commercial product contains some components in addition to the major components. The possible responsibility cf such component( $\mathrm{s}$ ) for transformation could not be neglected either.

Acknowledgement. This work was partly supported by a Grant-in-Aid for Scientific Research from the Ministry of Education, Japan.

\section{References}

1) Bloomfield, V. A., and Wilson, R. W. (1981): Polyamines in Biology and Medicine (eds. D. R. Molrris and L. J. Morton). vol. 8, Marcel Dekker, pp. 183-206.

2) Gfiller, E., Levy, C. C., and Russell, D. H. (1973): Polyamines in Normal and Neoplastic Growth (ed. D. H. Russell). Raven Press, pp. 335-342.

3) Hine, C. H. et al. (1958): Cancer Res., 18, 20-26.

4) Kano-Tanaka, K., and Tanaka, T. (1982) : Internat. J. Cancer, 30, 495-501.

5) Kano-Tanaka, K. et al. (1983): Proc. Jap. Cancer Assoc. 42nd Ann. Meet. (Nagoya), p. 56 .

6) Kato, K. et al. (1981): J. Neurochem., 36, 793-797.

7) Kato, T. et al. (1979) : Biochem. biophys. Acta (Amst.), 579, 216-227.

8) Kato, T. et al. (1984): Brain Res., 301, 83-93.

9) Laerum, O. D., and Rajewsky, M. F.'(1975) : J. Natl. Cancer Inst., 55, 1177-1187.

10) Oppenheimer, B. S. et al. (1953) : Science, 118, 305-306.

11) Scalabrino, G., and Ferioli, M. E. (1981): Adv. Cancer Res. (eds. G. Klein and S. Weinhouse). vol. 35, Acadelmic Press, pp. 151-268.

12) Sogin, D. C. (1976): J. Neurochem., 27, 1333-1337.

13) White, H. B., III, and Kaplan, N. (1969)': J. Biol. Chem., 244, 6031-6039. 